\title{
Gaussian and sparse processes are limits of generalized Poisson processes
}

\author{
Julien Fageot*, Virginie Uhlmann, Michael Unser \\ Biomedical Imaging Group, Ecole polytechnique fédérale de Lausanne (EPFL), Station 17, \\ CH-1015, Lausanne, Switzerland
}

\section{A R T I C L E I N F O}

\section{Article history:}

Received 11 October 2017

Received in revised form 18 July 2018

Accepted 9 October 2018

Available online 23 October 2018

Communicated by W.R. Madych

\section{Keywords:}

Sparse stochastic processes

Compound-Poisson processes

L-splines

Generalized random processes

Infinite divisibility

\begin{abstract}
A B S T R A C T
\end{abstract}
The theory of sparse stochastic processes offers a broad class of statistical models to study signals, far beyond the more classical class of Gaussian processes. In this framework, signals are represented as realizations of random processes that are solution of linear stochastic differential equations driven by Lévy white noises. Among these processes, generalized Poisson processes based on compound-Poisson noises admit an interpretation as random L-splines with random knots and weights. We demonstrate that every generalized Lévy process - from Gaussian to sparse - can be understood as the limit in law of a sequence of generalized Poisson processes. This enables a new conceptual understanding of sparse processes and suggests simple algorithms for the numerical generation of such objects.

(c) 2018 Elsevier Inc. All rights reserved.

\section{Introduction}

In their landmark paper on linear prediction [1], H.W. Bode and C.E. Shannon proposed that "a noise can be thought of as made up of a large number of closely spaced and very short impulses." In this work, we formulate this intuitive interpretation in a mathematically rigorous way. This allows us to extend this intuition beyond noise and to draw additional properties for the class of stochastic processes that can be linearly transformed into a white noise. More precisely, we show that the law of these processes can be approximated as closely as desired by generalized Poisson processes, which can also be viewed as random L-splines.

\subsection{Main result}

Let us define the first ingredient of our work. Splines are continuous-domain functions characterized by a sequence of knots and sample values. They provide a powerful framework to build discrete descriptions

\footnotetext{
* Corresponding author.

E-mail addresses: julien.fageot@epfl.ch (J. Fageot), virginie.uhlmann@epfl.ch (V. Uhlmann), michael.unser@epfl.ch (M. Unser).
} 


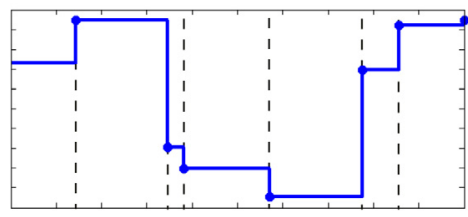

(a)

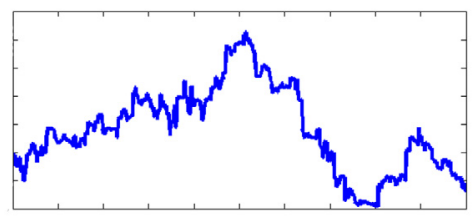

(c)

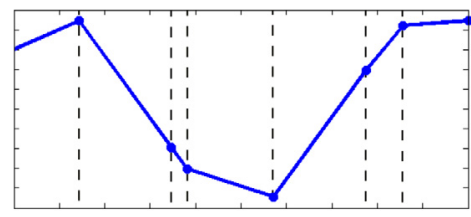

(b)

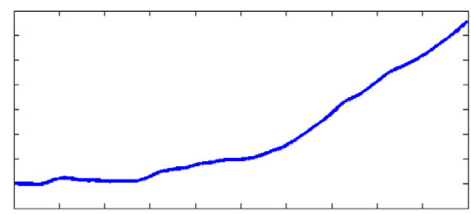

(d)

Fig. 1. Examples of deterministic splines: (a) piecewise constant, (b) piecewise linear; and of random processes: (c) Brownian motion, (d) second-order Gaussian process.

of continuous objects in sampling theory [2]. Initially defined as piecewise-polynomial functions [3], they were further generalized, starting from their connection with differential operators [4-6]. Let L be a suitable linear differential operator such as the derivative. Then, the function $s: \mathbb{R}^{d} \rightarrow \mathbb{R}$ is a non-uniform L-spline if

$$
\mathrm{L} s=\sum_{k=0}^{\infty} a_{k} \delta\left(\cdot-\boldsymbol{x}_{k}\right)
$$

is a sum of weighted and shifted Dirac impulses. The $a_{k}$ are the weights and the $\boldsymbol{x}_{k}$ the knots of the spline. Deterministic splines associated to various differential operators are depicted in Fig. 1a and 1b. Note that the knots $\boldsymbol{x}_{k}$ and weights $a_{k}$ can also be random, yielding stochastic splines.

The second main ingredient is a generative model of stochastic processes. Specifically, we consider linear differential equations of the form

$$
\mathrm{L} s=w,
$$

where $\mathrm{L}$ is a differential operator called the whitening operator and $w$ is a $d$-dimensional Lévy noise or innovation. Examples of such stochastic processes are illustrated in Fig. 1c and 1d.

Our goal in this paper is to build a bridge between linear stochastic differential equations (SDE) and splines. By comparing (1) and (2), one easily realizes that the differential operator L connects the random and deterministic frameworks. The link is even stronger when one notices that compound-Poisson white noises can be written as $w_{\text {Poisson }}=\sum_{k=0}^{\infty} a_{k} \delta\left(\cdot-\boldsymbol{x}_{k}\right)$ [7]. This means that the random processes that are solution of L $s=w_{\text {Poisson }}=\sum_{k=0}^{\infty} a_{k} \delta\left(\cdot-\boldsymbol{x}_{k}\right)$ are (random) L-splines.

Our main result thus uncovers the link between splines and random processes through the use of Poisson processes. A Poisson noise is made of a sparse sequence of weighted impulses whose jumps follow a common law. The average density of impulses $\lambda$ is the primary parameter of such a Poisson white noise. Upon increasing $\lambda$, one increases the average number of impulses by unit of time. Meanwhile, the intensity of the impulses is governed by the common law of the jumps of the noise. Upon decreasing this intensity, one makes the weights of the impulses smaller. By combining these two effects, the intuitive conceptualization of a white noise proposed by Bode and Shannon in [1] can be recovered.

Theorem 1. Every random process s solution of (2) is the limit in law of a sequence $\left(s_{n}\right)$ of generalized Poisson processes driven by compound-Poisson white noises and whitened by $\mathrm{L}$. 
We shall see that the convergence procedure is based on a coupled increase of the average density and a decrease of the intensity of the impulses of the Poisson noises. This is in the spirit of Bode and Shannon's quote and is, in fact, true for any Lévy noise.

\subsection{Motivations and connection to related works}

Our motivation to study stochastic differential equations such as (2) comes from signal processing. Random processes and random fields are notorious tools to model uncertainty and statistics of signals. Gaussian processes are by far the most studied stochastic models because of their fundamental properties (e.g., stability, finite variance, central-limit theorem) and their relative ease of use. They are the principal actors within the classical paradigm of statistical signal processing [8]. Many fractal-type signals are modeled as self-similar Gaussian processes [9-12].

However, lots of real-world signals are empirically observed to be inherently sparse, a property that is incompatible with Gaussianity [13-15]. In order to overcome the limitations of Gaussian model, several other stochastic models have been proposed for the study of sparse signals. They include infinite-variance $[12,16$, 17] or piecewise-constant models based on compound Poisson laws [7]. Interestingly, the compound Poisson processes, which are playing a crucial role in Theorem 1, are shown to be sparsest among the family of Lévy processes in the sense of approximation theory [18] and of information theory [19].

In this paper, we model signals as continuous-domain random processes defined over $\mathbb{R}^{d}$ that are solutions of a differential equation driven by Lévy noise. They are called generalized Lévy processes. We thus follow the general approach of [20] which includes the models mentioned above. The common feature of these processes is that their probability distributions are always infinitely divisible, meaning that they can be decomposed as sums of any length of independent and identically distributed random variables. Infinite divisibility is a key concept of continuous-domain random processes [21] and will be at the heart of our work. In order to embrace the largest variety of random models, we rely on the framework of generalized random processes, which is the probabilistic version of the theory of generalized functions of L. Schwartz [22]. Initially introduced independently by K. Itô [23] and I. Gelfand [24], the framework has been developed extensively by these two authors in [25] and [26]. A thrilling aspect of generalized Lévy processes is their ability to model sparse signals, as soon as the underlying white noise is non-Gaussian. This has been demonstrated both experimentally [27] and theoretically [18,28]. For this reason, the non-Gaussian members of the Lévy family are referred to as sparse stochastic processes $[20,29]$ to reflect their very compressibility nature.

Several behaviors can be observed within the family of generalized Lévy processes. For instance, selfsimilar Gaussian processes exhibit fractal behaviors. In one dimension, they include the fractional Brownian motion [11] and its higher-order extensions [30]. In higher dimensions, our framework covers the family of fractional Gaussian fields [31-34] and finite-variance self-similar fields that appear to converge to fractional Gaussian fields at large scales $[35,36]$. The self-similarity property is also compatible with the family of $\alpha$-stable processes [37] or fields [38], which have unbounded variances (when being non-Gaussian). More generally, every process considered in our framework is part of the Lévy family, including Laplace processes [39] and Student's processes [40]. Upon varying the operator L, one recovers Lévy processes [41], CARMA processes [42,43], and their multivariate generalizations $[44,45,20]$. Unlike those examples, the compound-Poisson processes, although members of the Lévy family, are piecewise-constant and have a finite rate of innovation (FRI) in the sense of [46]. For a signal, being FRI means that a finite quantity of information is sufficient to reconstruct it over a bounded domain.

The present paper is an extension of our previous contribution [47]. ${ }^{1}$ We believe that Theorem 1 is relevant for the conceptualization of random processes that are solution of linear SDE. Starting from the L-spline

\footnotetext{
${ }^{1}$ In this preliminary work, we had restricted our study to the family of CARMA Lévy processes in dimension $d=1$ and showed that they are limit in law of CARMA Poisson processes. Here, we extend our preliminary result in several ways. First, the class of processes we study now is much more general since we consider arbitrary operators. Moreover, we include multivariate random
} 
interpretation of generalized Poisson processes, the statistics of a more general process can be understood as a limit of the statistics of random L-splines. In general, the studied processes that are solution of (2) do not have a finite rate of innovation, except if the underlying white noise is Poisson. The convergence result helps us understand why non-Poisson processes do not have a finite rate of innovation. They in fact correspond to infinitely many impulses per unit of time as they can be approximated by FRI processes with an increasing and asymptotically infinite rate of innovation.

Interesting connections can also be drawn with some classical finite-dimension convergence results in probability theory. As mentioned earlier, there is a direct correspondence between Lévy white noises and infinitely divisible random variables. It is well known that any infinitely divisible random variable is the limit in law of a sequence of compound-Poisson random variables [21, Corollary 8.8]. Theorem 1 is the generalization of this result from real random variables to random processes that are solution of a linear SDE.

\subsection{Outline}

The paper is organized as follows: In Sections 2 and 3, we introduce the concepts of L-splines and generalized Lévy processes, respectively. A special emphasis is put on generalized Poisson processes in Section 4 as they embrace both generalized Lévy processes and (random) L-splines. Our main contribution is Theorem 1, which is proven in Section 5. Section 6 contains illustrative examples in the one- and two-dimensional settings, followed by concluding remarks in Section 7 .

\section{Nonuniform L-splines}

We denote by $\mathcal{S}\left(\mathbb{R}^{d}\right)$ the space of rapidly decaying functions from $\mathbb{R}^{d}$ to $\mathbb{R}$, that is, the space of functions that decay faster than any polynomial together with their derivatives. Its topological dual is $\mathcal{S}^{\prime}\left(\mathbb{R}^{d}\right)$, the Schwartz space of tempered generalized function [22]. We denote by $\langle u, \varphi\rangle$ the duality product between $u \in \mathcal{S}^{\prime}\left(\mathbb{R}^{d}\right)$ and $\varphi \in \mathcal{S}\left(\mathbb{R}^{d}\right)$. A linear and continuous operator L from $\mathcal{S}\left(\mathbb{R}^{d}\right)$ to $\mathcal{S}^{\prime}\left(\mathbb{R}^{d}\right)$ is spline-admissible if

- it is shift-invariant, meaning that

$$
\mathrm{L}\left\{\varphi\left(\cdot-\boldsymbol{x}_{0}\right)\right\}=\mathrm{L}\{\varphi\}\left(\cdot-\boldsymbol{x}_{0}\right)
$$

for every $\varphi \in \mathcal{S}\left(\mathbb{R}^{d}\right)$ and $\boldsymbol{x}_{0} \in \mathbb{R}^{d}$; and

- there exists a measurable function of slow growth (bounded by a polynomial) $\rho_{\mathrm{L}}$ such that

$$
\mathrm{L}\left\{\rho_{\mathrm{L}}\right\}=\delta
$$

with $\delta$ the Dirac delta function. The function $\rho_{\mathrm{L}}$ is a Green's function of L.

Definition 1. Let L be a spline-admissible operator with measurable Green's function $\rho_{\mathrm{L}}$. A nonuniform L-spline with knots $\left(\boldsymbol{x}_{k}\right)$ and weights $\left(a_{k}\right)$ is a function $s$ such that

$$
\mathrm{L} s=\sum_{k=0}^{\infty} a_{k} \delta\left(\cdot-\boldsymbol{x}_{k}\right) .
$$

processes, often called random fields. Finally, our preliminary report contained a mere sketch of the proof of [47, Theorem 8], while the current work is complete in this respect. 
Table 1

Some families of spline-admissible operators

\begin{tabular}{llll}
\hline Operator & Parameter & $\rho_{\mathrm{L}}(\boldsymbol{x})$ & Spline type \\
\hline $\mathrm{D}^{N}$ & $N \in \mathbb{N} \backslash\{0\}$ & $\frac{1}{(N-1) !} x^{N-1} u(x)$ & B-splines [2,3] \\
$(\mathrm{D}+\alpha \mathrm{I})$ & $\alpha \in \mathbb{C}, \Re(\alpha)>0$ & $\mathrm{e}^{-\alpha x} u(x)$ & E-splines [48] \\
$\mathrm{D}^{\gamma}$ & $\gamma>0$ & $\frac{1}{\Gamma(\gamma)} x^{\gamma-1} u(x)$ & fractional splines [6,49] \\
$\mathrm{D}_{x_{1}} \cdots \mathrm{D}_{x_{d}}$ & - & $u(\boldsymbol{x})=\prod_{i=1}^{d} u\left(x_{i}\right)$ & separable splines [20] \\
$(-\Delta)^{m / 2}$ & $m-d \in 2 \mathbb{N}$ & $c_{m, d}\|\boldsymbol{x}\|^{m-d} \log \|\boldsymbol{x}\|$ & cardinal polyharmonic splines [4] \\
$(-\Delta)^{\gamma / 2}$ & $\gamma-d \in \mathbb{R}^{+} \backslash 2 \mathbb{N}$ & $c_{\gamma, d}\|\boldsymbol{x}\|^{\gamma-d}$ & fractional polyharmonic splines [50] \\
\hline
\end{tabular}

Definition 1 implies that the generic expression for a nonuniform L-spline is

$$
s=p_{0}+\sum_{k \in \mathbb{Z}} a_{k} \rho_{\mathrm{L}}\left(\cdot-\boldsymbol{x}_{k}\right)
$$

with $p_{0}$ in the null space of $\mathrm{L}$ (i.e., $\left.\mathrm{L} p_{0}=0\right)$. Indeed, we have, by linearity and shift-invariance of $\mathrm{L}$, that

$$
\mathrm{L}\left\{s-\sum_{k \in \mathbb{Z}} a_{k} \rho_{\mathrm{L}}\left(\cdot-\boldsymbol{x}_{k}\right)\right\}=\mathrm{L} s-\sum_{k \in \mathbb{Z}} a_{k} \delta\left(\cdot-\boldsymbol{x}_{k}\right)=0 .
$$

Therefore, $\left(s-\sum_{k \in \mathbb{Z}} a_{k} \rho_{\mathrm{L}}\left(\cdot-\boldsymbol{x}_{k}\right)\right)$ is in the null space of $\mathrm{L}$.

We summarize in Table 1 important families of operators with their corresponding Green's function and the associated family of L-splines. The Heaviside function is denoted by $u$. The large variety of proposed splines, both in the univariate $(d=1)$ or multivariate contexts, illustrates the generality of our result.

\section{Generalized Lévy processes}

In this section, we briefly introduce the main tools and concepts for the characterization of Gaussian and sparse stochastic processes. For a more comprehensive description, we refer the reader to [20]. First, let us recall that a real random variable $X$ is a measurable function from a probability space $(\Omega, \mathcal{A}, \mathscr{P})$ to $\mathbb{R}$, endowed with the Borelian $\sigma$-field. The law of $X$ is the probability measure on $\mathbb{R}$ such that $\mathscr{P}_{X}([a, b])=$ $\mathscr{P}(a \leq X(\omega) \leq b)$. The characteristic function of $X$ is the (conjugate) Fourier transform of $\mathscr{P}$. For $\xi \in \mathbb{R}$, it is

$$
\widehat{\mathscr{P}}_{X}(\xi)=\int_{\mathbb{R}} \mathrm{e}^{\mathrm{i} \xi x} \mathrm{~d} \mathscr{P}_{X}(x)=\mathbb{E}\left[\mathrm{e}^{\mathrm{i} X \xi}\right]
$$

\subsection{Generalized random processes}

Generalized Lévy processes are defined in the framework of generalized random processes [25], which is the stochastic counterpart of the theory of generalized functions. For the purpose of this paper, a self-contained presentation of all the mathematical foundations together with the proofs of the results thereafter can be found in [51].

\subsubsection{Random elements in $\mathcal{S}^{\prime}\left(\mathbb{R}^{d}\right)$}

We first define the cylindrical $\sigma$-field on the Schwartz space $\mathcal{S}^{\prime}\left(\mathbb{R}^{d}\right)$, denoted by $\mathcal{B}_{c}\left(\mathcal{S}^{\prime}\left(\mathbb{R}^{d}\right)\right)$, as the $\sigma$-field generated by the cylinders

$$
\left\{v \in \mathcal{S}^{\prime}\left(\mathbb{R}^{d}\right), \quad\left(\left\langle v, \varphi_{1}\right\rangle, \ldots,\left\langle v, \varphi_{N}\right\rangle\right) \in B\right\},
$$

where $N \geq 1, \varphi_{1}, \ldots, \varphi_{N} \in \mathcal{S}\left(\mathbb{R}^{d}\right)$, and $B$ is a Borelian subset of $\mathbb{R}^{N}$. 
Definition 2. A generalized random process is a measurable function

$$
s:(\Omega, \mathcal{A}) \rightarrow\left(\mathcal{S}^{\prime}\left(\mathbb{R}^{d}\right), \mathcal{B}_{c}\left(\mathcal{S}^{\prime}\left(\mathbb{R}^{d}\right)\right)\right)
$$

The law of $s$ is then the probability measure $\mathscr{P}_{s}$ on $\mathcal{S}^{\prime}\left(\mathbb{R}^{d}\right)$, image of $\mathscr{P}$ by $s$. The characteristic functional of $s$ is the Fourier transform of its probability law, defined for $\varphi \in \mathcal{S}\left(\mathbb{R}^{d}\right)$ by

$$
\widehat{\mathscr{P}}_{s}(\varphi)=\int_{\mathcal{S}^{\prime}\left(\mathbb{R}^{d}\right)} \mathrm{e}^{\mathrm{i}\langle v, \varphi\rangle} \mathrm{d} \mathscr{P}_{s}(v)=\mathbb{E}\left[\mathrm{e}^{\mathrm{i}\langle s, \varphi\rangle}\right] .
$$

A generalized process $s$ is therefore a random element in $\mathcal{S}^{\prime}\left(\mathbb{R}^{d}\right)$. In particular, we have that

- for every $\omega \in \Omega$, the functional $\varphi \mapsto\langle s(\omega), \varphi\rangle$ is in $\mathcal{S}^{\prime}\left(\mathbb{R}^{d}\right)$; and

- for every $\varphi_{1}, \ldots \varphi_{N} \in \mathcal{S}\left(\mathbb{R}^{d}\right)$,

$$
\omega \mapsto \boldsymbol{Y}=\left(\left\langle s(\omega), \varphi_{1}\right\rangle, \ldots,\left\langle s(\omega), \varphi_{N}\right\rangle\right)
$$

is a random vector whose characteristic function is

$$
\widehat{\mathscr{P}}_{\boldsymbol{Y}}(\boldsymbol{\xi})=\widehat{\mathscr{P}}_{s}\left(\xi_{1} \varphi_{1}+\cdots+\xi_{N} \varphi_{N}\right)
$$

for every $\boldsymbol{\xi}=\left(\xi_{1}, \ldots, \xi_{N}\right) \in \mathbb{R}^{N}$.

The probability density functions (pdfs) of the random vectors $\boldsymbol{Y}$ in (12) are the finite-dimensional marginals of $s$. We shall omit the reference to $\omega \in \Omega$ thereafter.

\subsubsection{Abstract nuclear spaces}

We recall that function spaces are locally convex spaces, generally infinite-dimensional, whose elements are functions. To quote A. Pietsch in [52]: "The locally convex spaces encountered in analysis can be divided into two classes. First, there are the normed spaces (...). The second class consists of the so-called nuclear locally convex spaces." Normed spaces and nuclear spaces are mutually exclusive in infinite dimension [53, Corollary 2, pp. 520]. The typical example of nuclear function space is the Schwartz space $\mathcal{S}\left(\mathbb{R}^{d}\right)[53$, Corollary, pp. 530]; see also [26].

The theory of nuclear spaces was introduced by A. Grothendieck in [54]. The required formalism is more demanding than the simpler theory of Banach spaces. The payoff is that fundamental results of finite-dimensional probability theory can be directly extended to nuclear spaces, while such generalizations are not straightforward for Banach spaces.

Let $\mathcal{N}$ be a nuclear space and $\mathcal{N}^{\prime}$ its topological dual. As we did for $\mathcal{S}^{\prime}\left(\mathbb{R}^{d}\right)$ in Section 3.1.1, we define a generalized random process on $\mathcal{N}^{\prime}$ as a random variable $s$ from $\Omega$ to $\mathcal{N}^{\prime}$, endowed with the cylindrical $\sigma$-field $\mathcal{B}_{c}\left(\mathcal{N}^{\prime}\right)$. The law of $s$ is the image of $\mathscr{P}$ by $s$ and is a probability measure on $\mathcal{N}^{\prime}$. The characteristic functional of $s$ is $\widehat{\mathscr{P}}_{s}(\varphi)=\mathbb{E}\left[\mathrm{e}^{\mathrm{i}\langle s, \varphi\rangle}\right]$, defined for $\varphi \in \mathcal{N}$.

\subsubsection{Generalized Bochner and Lévy theorems}

First, we recall the two fundamental theorems that support the use of the characteristic function in probability theory.

Proposition 1 (Bochner theorem). A function $\widehat{\mathscr{P}}: \mathbb{R} \rightarrow \mathbb{C}$ is the characteristic function of some random variable $X$ if and only if $\widehat{\mathscr{P}}$ is continuous, positive-definite, and satisfies

$$
\widehat{\mathscr{P}}(0)=1 .
$$


Proposition 2 (Lévy theorem). Let $\left(X_{n}\right)_{n \in \mathbb{N}}$ and $X$ be real random variables. The sequence $X_{n}$ converges in law to $X$ if and only if for all $\xi \in \mathbb{R}$

$$
\widehat{\mathscr{P}}_{X_{n}}(\xi) \underset{n \rightarrow \infty}{\longrightarrow} \widehat{\mathscr{P}}_{X}(\xi)
$$

where $\widehat{\mathscr{P}}_{X_{n}}$ and $\widehat{\mathscr{P}}_{X}$ are respectively the characteristic functions of $X_{n}$ and $X$.

The infinite-dimensional generalizations of Propositions 1 and 2 were achieved during the 60 s and the $70 \mathrm{~s}$, and are effective for nuclear spaces only. See the introduction of [55] for a general discussion on this subject.

Initially conjectured by Gelfand, the so-called Minlos-Bochner theorem was proved by Minlos [56] for the case of Fréchet spaces and by Fernique for the general case [57].

Theorem 2 (Minlos-Bochner theorem). Let $\mathcal{N}$ be a nuclear space. The functional $\widehat{\mathscr{P}}$ from $\mathcal{N}$ to $\mathbb{C}$ is the characteristic functional of a generalized random process $s$ on $\mathcal{N}^{\prime}$ if and only if $\widehat{\mathscr{P}}$ is continuous, positivedefinite, and satisfies

$$
\widehat{\mathscr{P}}(0)=1 .
$$

The generalization of the Lévy theorem for nuclear spaces was obtained in [57] and is not as widely known as it should be. A sequence $\left(s_{n}\right)_{n \in \mathbb{N}}$ of generalized random processes in $\mathcal{N}^{\prime}$ is said to converge in law to $s$, which we denote by $s_{n} \underset{n \rightarrow \infty}{\stackrel{(d)}{\longrightarrow}} s$, if the underlying probability measures $\widehat{\mathscr{P}}_{s_{n}}$ converge weakly to $\widehat{\mathscr{P}}_{s}$, in such a way that

$$
\int_{\mathcal{S}^{\prime}\left(\mathbb{R}^{d}\right)} f(v) \mathrm{d} \widehat{\mathscr{P}}_{s_{n}}(v) \underset{n \rightarrow \infty}{\longrightarrow} \int_{\mathcal{S}^{\prime}\left(\mathbb{R}^{d}\right)} f(v) \mathrm{d} \widehat{\mathscr{P}}_{s}(v)
$$

for any continuous bounded function $f: \mathcal{S}^{\prime}\left(\mathbb{R}^{d}\right) \rightarrow \mathbb{R}$.

Theorem 3 (Fernique-Lévy theorem). Let $\mathcal{N}$ be a nuclear space. Let $\left(s_{n}\right)_{n \in \mathbb{N}}$ and $s$ be generalized random processes on $\mathcal{N}^{\prime}$. Then, $s_{n} \underset{n \rightarrow \infty}{\stackrel{(d)}{\longrightarrow}} s$ if and only if the underlying characteristic functionals of $s_{n}$ converge pointwise to the characteristic functional of $s$, so that

$$
\widehat{\mathscr{P}}_{s_{n}}(\varphi) \underset{n \rightarrow \infty}{\longrightarrow} \widehat{\mathscr{P}}_{s}(\varphi)
$$

for all $\varphi \in \mathcal{N}$.

Interestingly, it also appears that nuclear spaces are the unique Fréchet spaces for which the Lévy theorem still holds [58, Theorem 5.3]. Therefore, the nuclearity is at the heart of infinite-dimensional generalization of Lévy theorem.

We shall use Theorems 2 and 3 with $\mathcal{N}=\mathcal{S}\left(\mathbb{R}^{d}\right)$. Theorem 2 is our main tool to construct solutions of stochastic differential equations as generalized random processes. On the other hand, Theorem 3 allows one to show the convergence in law of a family of generalized random processes.

\subsection{Lévy white noises and generalized Lévy processes}

White noises can only be defined as generalized random processes, since they are too erratic to be defined as classical, pointwise processes. 


\subsubsection{Lévy exponents}

Lévy white noises are in a one-to-one correspondence with infinitely divisible random variables. A random variable $X$ is said to be infinitely divisible if it can be decomposed for every $N \geq 1$ as

$$
X=X_{1}+\cdots+X_{N}
$$

where the $X_{n}$ are independent and identically distributed (i.i.d.). The characteristic function of an infinitely divisible law has the particularity of having no zero [21, Lemma 7.5], and therefore can be written as $\widehat{\mathscr{P}}_{X}(\xi)=\exp (f(\omega))$ with $f$ a continuous function [21, Lemma 7.6].

Definition 3. A Lévy exponent is a function $f: \mathbb{R} \rightarrow \mathbb{C}$ that is the continuous log-characteristic function of an infinitely divisible law.

Theorem 4 gives the fundamental decomposition of a Lévy exponent. It is proved in [21, Section 8].

Theorem 4 (Lévy-Khintchine theorem). A function $f: \mathbb{R} \rightarrow \mathbb{C}$ is a Lévy exponent if and only if it can be written as

$$
f(\xi)=\mathrm{i} \mu \xi-\frac{\sigma^{2} \xi^{2}}{2}+\int_{\mathbb{R}}\left(\mathrm{e}^{\mathrm{i} \xi t}-1-\mathrm{i} \xi t 1_{|t| \leq 1}\right) V(\mathrm{~d} t),
$$

where $\mu \in \mathbb{R}, \sigma^{2} \geq 0$, and $V$ is a Lévy measure, which is a measure on $\mathbb{R}$ with

$$
\int_{\mathbb{R}} \min \left(1, t^{2}\right) V(\mathrm{~d} t)<\infty \text { and } V(\{0\})=0 .
$$

We call $\left(\mu, \sigma^{2}, V\right)$ the Lévy triplet associated to $f(\xi)$. If, moreover, one has that

$$
\int_{|t| \geq 1}|t|^{\epsilon} V(\mathrm{~d} t)<\infty
$$

for some $\epsilon>0$, then $V$ is called a Lévy-Schwartz measure and one says that $f$ satisfies the Schwartz condition.

\subsubsection{Lévy white noises}

If $f$ is a Lévy exponent satisfying the Schwartz condition, then the functional

$$
\varphi \mapsto \exp \left(\int_{\mathbb{R}^{d}} f(\varphi(\boldsymbol{x})) \mathrm{d} \boldsymbol{x}\right)
$$

is a valid characteristic functional on $\mathcal{S}\left(\mathbb{R}^{d}\right)$ [59, Theorem 3]. Hence, as a consequence of Theorem 2, there exists a generalized random process having this characteristic functional.

Definition 4. A Lévy white noise on $\mathcal{S}^{\prime}\left(\mathbb{R}^{d}\right)$ is the generalized random process $w$ whose characteristic functional has the form

$$
\widehat{\mathscr{P}}_{w}(\varphi)=\exp \left(\int_{\mathbb{R}^{d}} f(\varphi(\boldsymbol{x})) \mathrm{d} \boldsymbol{x}\right)
$$

where $f$ is a Lévy exponent satisfying the Schwartz condition. 
Lévy white noises are stationary, meaning that $w\left(\cdot-\boldsymbol{x}_{0}\right)$ and $w$ have the same probability law for every $\boldsymbol{x}_{0}$. They are, moreover, independent at every point, in the sense that $\left\langle w, \varphi_{1}\right\rangle$ and $\left\langle w, \varphi_{2}\right\rangle$ are independent if $\varphi_{1}$ and $\varphi_{2}$ have disjoint supports.

\subsubsection{Generalized Lévy processes}

We want to define random processes $s$ solutions of the equation $\mathrm{L} s=w$. This requires one to identify compatibility conditions between $\mathrm{L}$ and $w$. This question was addressed in previous works $[20,59,60]$ that we summarize now.

Definition 5. Let $\left(\mu, \sigma^{2}, V\right)$ be a Lévy triplet. For $0 \leq p_{\min } \leq p_{\max } \leq 2$, one says that $\left(\mu, \sigma^{2}, V\right)$ is a $\left(p_{\min }, p_{\max }\right)$-triplet if there exists

$$
p_{\min } \leq p \leq q \leq p_{\max }
$$

such that

1. $\int_{|t| \geq 1}|t|^{p} V(\mathrm{~d} t)<\infty$,

2. $\int_{|t|<1}|t|^{q} V(\mathrm{~d} t)<\infty$,

3. $p_{\text {min }}=\inf (p, 1)$ if $V$ is non-symmetric or $\mu \neq 0$, and

4. $p_{\max }=2$ if $\sigma^{2} \neq 2$.

If $f$ is the Lévy exponent associated to $\left(\mu, \sigma^{2}, V\right)$, then one also says that $f$ is a $\left(p_{\min }, p_{\max }\right)$-exponent.

If $V$ is symmetric, then $(0,0, V)$ is a $\left(p_{\min }, p_{\max }\right)$-triplet if and only if

$$
\int_{|t| \geq 1}|t|^{p_{\min }} V(\mathrm{~d} t) \text { and } \int_{|t|<1}|t|^{p_{\max }} V(\mathrm{~d} t)<\infty .
$$

The other conditions are added to deal with the presence of a Gaussian part (for which $p_{\max }=2$ ) and the existence of asymmetry (for which $p_{\min } \geq 1$ ). Note, moreover, that every Lévy exponent is a $(0,2)$-exponent and that a Lévy exponent satisfies the Schwartz condition if and only if it is an $(\epsilon, 2)$-exponent for some $0<\epsilon \leq 2$.

Definition 6. Let L be a spline-admissible operator and $w$ a Lévy white noise with Lévy exponent $f$. One says that $(\mathrm{L}, w)$ is compatible if there exists

$$
0<p_{\min } \leq p_{\max } \leq 2
$$

such that

- the function $f$ is a $\left(p_{\min }, p_{\max }\right)$-exponent; and

- the adjoint $\mathrm{L}^{*}$ of $\mathrm{L}$ admits a left inverse $\mathrm{T}$ such that

$$
\mathrm{TL}^{*}\{\varphi\}=\varphi, \quad \forall \varphi \in \mathcal{S}\left(\mathbb{R}^{d}\right)
$$

is linear and continuous from $\mathcal{S}\left(\mathbb{R}^{d}\right)$ to $L_{p_{\min }}\left(\mathbb{R}^{d}\right) \cap L_{p_{\max }}\left(\mathbb{R}^{d}\right)$.

We know especially that, if $(\mathrm{L}, w)$ is compatible, then the functional $\varphi \mapsto \widehat{\mathscr{P}}_{w}(\mathrm{~T}\{\varphi\})$ is a valid characteristic functional on $\mathcal{S}\left(\mathbb{R}^{d}\right)$ [59, Theorem 5]. Hence, there exists a generalized random process $s$ with $\widehat{\mathscr{P}}_{s}(\varphi)=\widehat{\mathscr{P}}_{w}(\mathrm{~T}\{\varphi\})$. Moreover, we have by duality that $\langle\mathrm{L} s, \varphi\rangle=\left\langle s, \mathrm{~L}^{*} \varphi\right\rangle$ and, therefore, that 


$$
\widehat{\mathscr{P}}_{\mathrm{L} s}(\varphi)=\widehat{\mathscr{P}}_{s}\left(\mathrm{~L}^{*}\{\varphi\}\right)=\widehat{\mathscr{P}}_{w}\left(\mathrm{TL}^{*}\{\varphi\}\right)=\widehat{\mathscr{P}}_{w}(\varphi)
$$

or, equivalently, that $\mathrm{L} s \stackrel{(d)}{=} w$. When $(\mathrm{L}, w)$ is compatible, we formally denote it by

$$
s=\mathrm{L}^{-1} w
$$

which implicitly means that we fix an operator $\mathrm{T}$ satisfying the conditions of Definition 6 and that the characteristic functional of $s$ is $\varphi \mapsto \widehat{\mathscr{P}}_{w}(\mathrm{~T}\{\varphi\})$.

Definition 7. Let $(\mathrm{L}, w)$ be compatible. The process $s=\mathrm{L}^{-1} w$ is called a generalized Lévy process.

The family of generalized Lévy processes includes generalized Gaussian processes for which the underlying white noise is Gaussian. As explained in the introduction, the non-Gaussian members of the family appears to be more compressible than their Gaussian counterpart, and are called sparse stochastic processes for this reason [20]. Our analysis however applies for both Gaussian and sparse processes, and we shall mostly refer to the broad family of generalized Lévy processes, which includes both of them, in our results.

The inequality of Proposition 3 will be useful in the sequel.

Proposition 3 (Corollary 1, [59]). Let $f$ be a ( $\left.p_{\min }, p_{\max }\right)$-exponent with $0<p_{\min } \leq p_{\max } \leq 2$. Then, there exist constants $\nu_{1}, \nu_{2}>0$ such that, for every $\xi \in \mathbb{R}$,

$$
|f(\xi)| \leq \nu_{1}|\xi|^{p_{\min }}+\nu_{2}|\xi|^{p_{\max }}
$$

Strictly speaking, Corollary 1 in [59] states that the non-Gaussian part of $f$, denoted by $g=f(\xi)-\mathrm{i} \mu \xi+$ $\frac{\sigma^{2} \xi^{2}}{2}$, satisfies

$$
|g(\xi)| \leq \kappa_{1}|\xi|^{p_{\min }}+\kappa_{2}|\xi|^{p_{\max }}
$$

for some constants $\kappa_{1}, \kappa_{2}>0$. We easily propagate this inequality to $f$ by exploiting that $p_{\min } \leq 1\left(p_{\max }=2\right.$, respectively) when $\mu \neq 0\left(\sigma^{2} \neq 0\right.$, respectively).

Proposition 3 allows us to extend the domain of continuity $\widehat{\mathscr{P}}_{w}(\varphi)$ from $\mathcal{S}\left(\mathbb{R}^{d}\right)$ to $L_{p_{\min }}\left(\mathbb{R}^{d}\right) \cap L_{p_{\max }}\left(\mathbb{R}^{d}\right)$. Indeed, (31) implies that

$$
\left|\log \widehat{\mathscr{P}}_{w}(\varphi)\right| \leq \int_{\mathbb{R}^{d}}|f(\varphi(\boldsymbol{x}))| \mathrm{d} \boldsymbol{x} \leq \nu_{1}\|\varphi\|_{p_{\min }}^{p_{\min }}+\nu_{2}\|\varphi\|_{p_{\max }}^{p_{\max }}
$$

Therefore, $\widehat{\mathscr{P}}_{w}$ is well-defined over $L_{p_{\min }}\left(\mathbb{R}^{d}\right) \cap L_{p_{\max }}\left(\mathbb{R}^{d}\right)$ and continuous at $\varphi=0$. Since characteristic functionals are positive-definite, the continuity at 0 implies the continuity over $L_{p_{\min }}\left(\mathbb{R}^{d}\right) \cap L_{p_{\max }}\left(\mathbb{R}^{d}\right)[61]$.

Corollary 1. With the notations of Proposition 3, the characteristic functional $\widehat{\mathscr{P}}_{w}(\varphi)$ of the Lévy white noise $w$ on $\mathcal{S}^{\prime}\left(\mathbb{R}^{d}\right)$ with Lévy exponent $f$, which is a priori defined for $\varphi \in \mathcal{S}\left(\mathbb{R}^{d}\right)$, can be extended continuously to $L_{p_{\text {min }}}\left(\mathbb{R}^{d}\right) \cap L_{p_{\max }}\left(\mathbb{R}^{d}\right)$.

\section{Generalized Poisson processes: a bridge between L-splines and generalized Lévy processes}

Generalized Poisson processes are generalized Lévy processes driven by impulsive noise. They can be interpreted as random L-splines, which makes them conceptually more accessible than other generalized Lévy processes. 
Definition 8. Let $\lambda>0$ and let $P$ be a probability law on $\mathbb{R} \backslash\{0\}$ such that there exists $\epsilon>0$ for which $\int_{\mathbb{R} \backslash\{0\}}|t|^{\epsilon} P(\mathrm{~d} t)<\infty$. The impulsive noise $w$ with rate $\lambda>0$ and amplitude probability law $P$ is the process with characteristic functional

$$
\widehat{\mathscr{P}}_{w}(\varphi)=\exp \left(\lambda \int_{\mathbb{R}^{d}} \int_{\mathbb{R}}\left(\mathrm{e}^{\mathrm{i} \varphi(\boldsymbol{x}) t}-1\right) P(\mathrm{~d} t) \mathrm{d} \boldsymbol{x}\right)
$$

According to [7, Theorem 1], one has that

$$
w=\sum_{n \in \mathbb{Z}} a_{n} \delta\left(\cdot-\boldsymbol{x}_{n}\right)
$$

where the sequence $\left(a_{n}\right)$ is i.i.d. with law $P$ and the sequence $\left(\boldsymbol{x}_{n}\right)$, independent of $\left(a_{n}\right)$, is such that, for every finite measure Borel set $A \subset \mathbb{R}^{d}, \operatorname{card}\left\{n \in \mathbb{Z}, \boldsymbol{x}_{n} \in A\right\}$ is a Poisson random variable with parameter $\lambda L(A), L$ being the Lebesgue measure on $\mathbb{R}^{d}$.

Proposition 4. An impulsive noise with rate $\lambda>0$ and jump-size probability law $P$ is a Lévy white noise with triplet $\left(\lambda \mu_{P}, 0, \lambda P\right)$, where $\mu_{P}=\int_{|t|<1} t P(\mathrm{~d} t)$. Moreover, its Lévy exponent is given by

$$
f(\xi)=\lambda(\widehat{P}(\xi)-1)
$$

with $\widehat{P}$ the characteristic function of $P$.

Proof. This result is obvious by comparing (34) with the general form of a Lévy exponent (20).

Definition 9. Let $(\mathrm{L}, w)$ be compatible with $w$ an impulsive noise. Then, the process $s=\mathrm{L}^{-1} w$ is called a generalized Poisson process.

Proposition 5. A generalized Poisson process $s$ is almost surely a nonuniform L-spline.

Proof. Let $s=\mathrm{L}^{-1} w$ be a generalized Poisson process, with $w$ an impulsive noise and L a spline-admissible operator. Then, according to (35), we have that

$$
\mathrm{L} s \stackrel{(d)}{=} w=\sum_{n \in \mathbb{Z}} a_{n} \delta\left(\cdot-\boldsymbol{x}_{n}\right)
$$

Based on Definition 1, the function $s$ is therefore an L-spline almost surely.

This connection with spline theory gives a very intuitive way of understanding generalized Poisson processes: their realizations are nonuniform L-splines.

\section{Generalized Lévy processes as limits of generalized Poisson processes}

This section is dedicated to the proof of Theorem 1. We start with some notations. The characteristic function of a compound-Poisson law with rate $\lambda$ and jump law $P$ is given by

$$
\mathrm{e}^{\lambda(\widehat{P}(\xi)-1)}
$$


with $\widehat{P}$ the characteristic function of $P$. If $f$ is a Lévy exponent, then one denotes by $P_{f}$ the compoundPoisson probability law with rate $\lambda=1$ and by law of jumps the infinitely divisible law with characteristic function $\mathrm{e}^{f}$. The characteristic function of $P_{f}$ is therefore $\widehat{P}_{f}(\xi)=\mathrm{e}^{\mathrm{e}^{f(\xi)}-1}$ and the Lévy exponent of $P_{f}$ is

$$
\mathrm{e}^{f(\xi)}-1
$$

\subsection{Compatibility of impulsive noises}

First of all, we show that, if an operator L is compatible with a Lévy noise $w$ whose Lévy exponent is $f$, then it is also compatible with any impulsive noise with the law of jumps $P_{f}$.

Proposition 6. If $f$ is a $\left(p_{\min }, p_{\max }\right)$-exponent, then, for every $\lambda>0$ and $\tau \neq 0$, the Lévy exponent

$$
f_{\lambda, \tau}(\xi)=\lambda\left(\mathrm{e}^{\tau f(\xi)}-1\right)
$$

associated with the generalized Poisson process of rate $\lambda$ and law of jumps $P_{\tau f}$ is also a $\left(p_{\min }, p_{\max }\right)$-exponent.

We shall make use of Lemma 1, which provides a result on infinitely divisible law and is proved in [21, Theorem 25.3].

Lemma 1. For $Z$ an infinitely divisible random variable with Lévy measure $V_{Z}$ and $0<p \leq 2$, we have the equivalence

$$
\mathbb{E}\left[|Z|^{p}\right]<\infty \Longleftrightarrow \int_{|t| \geq 1}|t|^{p} V_{Z}(\mathrm{~d} t)<\infty .
$$

Proof of Proposition 6. Note first that both $\tau f$ and $f_{\lambda, \tau}$ are Lévy exponents. Let $\left(\mu, \sigma^{2}, V\right)$ be the $\left(p_{\min }, p_{\max }\right)$-triplet associated with $f$. The Lévy triplet of $f_{\lambda, \tau}$ is

$$
\left(\lambda \mu_{P_{\tau f}}, 0, \lambda P_{\tau f}\right)
$$

where we recall that $P_{\tau f}$ is the compound-Poisson law with rate $\lambda=1$ and law of jumps corresponding to the infinitely divisible random variable with Lévy exponent $\tau f$. In addition,

$$
\mu_{P_{\tau f}}=\int_{0<|t|<1} t P_{\tau f}(\mathrm{~d} t) .
$$

Let $X$ (respectively, $Y$ ) be an infinitely divisible random variable with Lévy exponent $f\left(f_{\lambda, \tau}\right.$, respectively).

Let $\mu=\sigma^{2}=0$ and $V$ be symmetric. In this case, we have that $\mu_{P_{\tau f}}=0$ and $P_{\tau f}$ is symmetric, so that $f_{\lambda, \tau}$ is a $\left(p_{\min }, p_{\max }\right)$-exponent if and only if

$$
\begin{gathered}
\int_{|t| \geq 1}|t|^{p_{\min }} P_{\tau f}(\mathrm{~d} t)<\infty \\
\int_{0<|t|<1}|t|^{p_{\max }} P_{\tau f}(\mathrm{~d} t)<\infty .
\end{gathered}
$$

Because $P_{\tau f}$ is a probability measure, (45) is obvious. Based on Lemma 1, (44) is equivalent to the condition 


$$
\mathbb{E}\left[|Y|^{p_{\text {min }}}\right]<\infty
$$

The random variable $Y$ being compound-Poisson, we have that

$$
Y \stackrel{(d)}{=} \sum_{i=1}^{N} X_{i}
$$

with $N$ a Poisson random variable of parameter $\lambda$ and $\left(X_{i}\right)_{i \in \mathbb{N}}$ an i.i.d. vector with common law $P_{\tau f}$.

Let us fix $x, y \in \mathbb{R}$. If $0<p<1$, then we have that

$$
|x+y|^{p} \leq|x|^{p}+|y|^{p} .
$$

On the contrary, if $1 \leq p \leq 2$, then the inequality

$$
\left|\frac{x+y}{2}\right|^{p} \leq \frac{|x|^{p}+|y|^{p}}{2}
$$

follows from the convexity of $x \mapsto x^{p}$ on $\mathbb{R}^{+}$. From these two inequalities, we see that for any $0<p \leq 2$ and $\left(x_{i}\right)_{1 \leq i \leq N}$,

$$
\left|\sum_{i=1}^{N} x_{i}\right|^{p} \leq N^{\max (p-1,0)} \sum_{i=1}^{N}\left|x_{i}\right|^{p} \leq N \sum_{i=1}^{N}\left|x_{i}\right|^{p} .
$$

Therefore, we have that

$$
\begin{aligned}
\mathbb{E}\left[|Y|^{p_{\text {min }}}\right] & =\mathbb{E}\left[\left|\sum_{i=1}^{N} X_{i}\right|^{p_{\text {min }}}\right] \leq \mathbb{E}\left[N \sum_{i=1}^{N}\left|X_{i}\right|^{p_{\text {min }}}\right] \\
& =\sum_{n \geq 0} n \mathbb{P}(N=n) \mathbb{E}\left[\sum_{i=1}^{n}\left|X_{i}\right|^{p_{\text {min }}}\right]=\left(\sum_{n \geq 0} n^{2} \mathbb{P}(N=n)\right) \times \mathbb{E}\left[\left|X_{1}\right|^{p_{\text {min }}}\right] \\
& =\mathbb{E}\left[N^{2}\right] \times \mathbb{E}\left[\left|X_{1}\right|^{p_{\text {min }}}\right]<\infty
\end{aligned}
$$

This shows that $f_{\lambda, \tau}$ is a $\left(p_{\min }, p_{\max }\right)$-exponent.

General case. By assumption, $\left(\mu, \sigma^{2}, V\right)$ is a $\left(p_{\min }, p_{\max }\right)$-triplet, so that there exist $p, q$ such that $p_{\min } \leq$ $p \leq q \leq p_{\max }$ and

$$
\int_{|t| \geq 1}|t|^{p} V(\mathrm{~d} t)<\infty \text { and } \int_{|t|<1}|t|^{q} V(\mathrm{~d} t)<\infty .
$$

As we did for Case $a$ ), we deduce that

$$
\int_{|t| \geq 1}|t|^{p} P_{\tau f}(\mathrm{~d} t)<\infty \text { and } \int_{|t|<1}|t|^{q} P_{\tau f}(\mathrm{~d} t)<\infty .
$$

This means that the Lévy measure $P_{\tau f}$ of $f_{\lambda, \tau}$ satisfies the first and second conditions in Definition 5 . Moreover, if either $V$ is non-symmetric or $\mu \neq 0$, then either $P_{\tau f}$ is not symmetric or $\mu_{P_{\tau f}} \neq 0$. However, in this case $p_{\min } \leq 1$, so that the third condition in Definition 5 is satisfied. Similarly, if $\sigma^{2} \neq 0$, then $p_{\max }=2$ and the fourth condition in Definition 5 is satisfied. Hence, $f_{\lambda, \tau}$ is a $\left(p_{\min }, p_{\max }\right)$-exponent. 
Corollary 2. Let L be a spline-admissible operator and $w$ a Lévy white noise with Lévy exponent $f$. Then, $\mathrm{L}$ is compatible with any impulsive noise with rate $\lambda>0$ and jump-size law $P_{\tau f}$ for $\tau>0$.

Proof. Knowing that $f_{\lambda, \tau}$ is a $\left(p_{\min }, p_{\max }\right)$-exponent, we deduce from Definition 6 that $\left(\mathrm{L}, w_{\lambda, \tau}\right)$ is compatible, where $w_{\lambda, \tau}$ is the impulsive noise with Lévy exponent $f_{\lambda, \tau}$.

\subsection{Generalized Lévy processes as limits of generalized Poisson processes}

Lemma 2. Let $f$ be a $\left(p_{\min }, p_{\max }\right)$-exponent for some $0<p_{\min } \leq p_{\max } \leq 2$ and let $w$ be the associated Lévy white noise. Let $f_{n}$ be the Lévy exponent defined by

$$
f_{n}(\xi)=n\left(\mathrm{e}^{f(\xi) / n}-1\right)
$$

and let $w_{n}$ be the impulsive noise with exponent $f_{n}$. Then, for every $\varphi \in L_{p_{\min }}\left(\mathbb{R}^{d}\right) \cap L_{p_{\max }}\left(\mathbb{R}^{d}\right)$, we have that

$$
\widehat{\mathscr{P}}_{w_{n}}(\varphi) \underset{n \rightarrow \infty}{\longrightarrow} \widehat{\mathscr{P}}_{w}(\varphi)
$$

Proof. First of all, the function $f_{n}$ is the Lévy exponent associated to the compound-Poisson law with rate $n$ and jump-size law with Lévy exponent $f / n$. Let $\varphi \in L_{p_{\min }}\left(\mathbb{R}^{d}\right) \cap L_{p_{\max }}\left(\mathbb{R}^{d}\right)$. According to Corollary $1, \widehat{\mathscr{P}}_{w}(\varphi)$ is well-defined. From Proposition 6 (applied with $\lambda=1 / \tau=n$ ), we also know that $f_{n}$ is a $\left(p_{\min }, p_{\max }\right)$-exponent, so that $\widehat{\mathscr{P}}_{w_{n}}(\varphi)$ is also well-defined. We can now prove the convergence. For every fixed $\boldsymbol{x} \in \mathbb{R}^{d}$, we have that

$$
f_{n}(\varphi(\boldsymbol{x}))=n\left(\mathrm{e}^{f(\varphi(\boldsymbol{x})) / n}-1\right) \underset{n \rightarrow \infty}{\longrightarrow} f(\varphi(\boldsymbol{x}))
$$

The goal is now to show that

$$
\int_{\mathbb{R}^{d}} f_{n}(\varphi(\boldsymbol{x})) \mathrm{d} \boldsymbol{x} \underset{n \rightarrow \infty}{\rightarrow} \int_{\mathbb{R}^{d}} f(\varphi(\boldsymbol{x})) \mathrm{d} \boldsymbol{x}
$$

From (20), we readily see that

$$
\Re(f(\varphi(\boldsymbol{x})))=\int_{\mathbb{R}}(\cos (t \varphi(\boldsymbol{x}))-1) V(\mathrm{~d} t)-\frac{\sigma^{2} \varphi(\boldsymbol{x})^{2}}{2} \leq 0 .
$$

Let $x \leq 0$ and $y \in \mathbb{R}$. Due to the convexity of the exponential, $\left|\mathrm{e}^{x}-1\right| \leq|x|$. Moreover, $\left|\mathrm{e}^{\mathrm{i} y}-1\right|=$ $2|\sin (y / 2)| \leq|y|$. Thus, for $z=x+\mathrm{i} y$,

$$
\left|\mathrm{e}^{z}-1\right|=\left|\mathrm{e}^{\mathrm{i} y}\left(\mathrm{e}^{x}-1\right)+\mathrm{e}^{\mathrm{i} y}-1\right| \leq|x|+|y| \leq \sqrt{2}|z|
$$

We now apply (59) to $z=f(\varphi(\boldsymbol{x})) / n$, whose real part is negative due to (58), and deduce that

$$
\left|f_{n}(\varphi(\boldsymbol{x}))\right|=n\left|\mathrm{e}^{f(\varphi(\boldsymbol{x})) / n}-1\right| \leq n \sqrt{2}|f(\varphi(\boldsymbol{x}))| / n=\sqrt{2}|f(\varphi(\boldsymbol{x}))|
$$

The function $\boldsymbol{x} \mapsto|f(\varphi(\boldsymbol{x}))|$ is in $L_{1}\left(\mathbb{R}^{d}\right)$ according to Proposition 3 . We thus apply the Lebesgue dominatedconvergence theorem to deduce (57) and, as a consequence, (55) holds. 
Theorem 5. Let $(\mathrm{L}, w)$ be compatible and let $s=\mathrm{L}^{-1} w$. The Lévy exponent of $w$ is denoted by $f$. For $n \geq 1$, we set $w_{n}$ the impulsive noise with Lévy exponent $f_{n}$ defined in (54) and $s_{n}=\mathrm{L}^{-1} w_{n}$ the associated generalized Poisson process. Then,

$$
s_{n} \underset{n \rightarrow \infty}{\stackrel{(d)}{\longrightarrow}} s .
$$

We note that Theorem 5 is a reformulation-hence implies - Theorem 1, in which we explicitly state the way we approximate the process $s$ with generalized Poisson processes $s_{n}$.

Proof. We fix an operator $\mathrm{T}$ defined as a left inverse of $\mathrm{L}^{*}$ associated with the compatible couple (L, $\left.w\right)$ as in Definition 6. For every $n \geq 1,\left(\mathrm{~L}, w_{n}\right)$ is compatible by applying Corollary 2 with $\lambda=1 / \tau=n$. Hence, the process $s_{n}$ with characteristic functional $\widehat{\mathscr{P}}_{w_{n}}(\mathrm{~T}\{\varphi\})$ is well-defined for every $n$.

Then, for every $\varphi \in \mathcal{S}\left(\mathbb{R}^{d}\right)$, we have by compatibility that

$$
\mathrm{T}\{\varphi\} \in L_{p_{\min }}\left(\mathbb{R}^{d}\right) \cap L_{p_{\max }}\left(\mathbb{R}^{d}\right) .
$$

By applying Lemma 2 to $T\{\varphi\}$, we deduce that

$$
\widehat{\mathscr{P}}_{w_{n}}(\mathrm{~T}\{\varphi\}) \underset{n \rightarrow \infty}{\longrightarrow} \widehat{\mathscr{P}}_{w}(\mathrm{~T}\{\varphi\}) .
$$

For $\varphi \in \mathcal{S}\left(\mathbb{R}^{d}\right)$, we have therefore that

$$
\widehat{\mathscr{P}}_{s_{n}}(\varphi)=\widehat{\mathscr{P}}_{w_{n}}(\mathrm{~T}\{\varphi\}) \underset{n \rightarrow \infty}{\longrightarrow} \widehat{\mathscr{P}}_{w}(\mathrm{~T}\{\varphi\})=\widehat{\mathscr{P}}_{s}(\varphi) .
$$

Finally, Theorem 3 implies that

$$
s_{n} \underset{n \rightarrow \infty}{\stackrel{(d)}{\longrightarrow}} s .
$$

\section{Simulations}

Here, we illustrate the convergence result of Theorem 1 on generalized Lévy processes of three types, namely

- Gaussian processes based on Gaussian white noise, which are non-sparse;

- Laplace processes based on Laplace noise, which are sparse and have finite variance;

- Cauchy processes based on Cauchy white noise, our prototypical example of infinite-variance sparse model.

For a given white noise $w$ with Lévy exponent $f$, we consider compound-Poisson processes that follow the principle of Lemma 2. Therefore, we consider compound-Poisson white noises with parameter $\lambda$ and law of jumps with Lévy exponent $\frac{f}{\lambda}$, for increasing values of $\lambda$.

In Table 2, we specify the parameters and Lévy exponents of six types of noise: Gaussian, Laplace, Cauchy, and their corresponding compound-Poisson noises. We name a compound-Poisson noise in relation to the law of its jumps (e.g., the compound-Poisson noise with Gaussian jumps is called a Gauss-Poisson noise). As $\lambda$ increases, the associated compound-Poisson noise features more and more jumps on average ( $\lambda$ per unit of volume) and is more and more concentrated towards 0. For instance, in the Gaussian case, the Gauss-Poisson noise has jumps with variance $\frac{\sigma^{2}}{\lambda} \underset{\lambda \rightarrow \infty}{\longrightarrow} 0$. To illustrate our results, we provide simulations for the 1-D and 2-D settings. 
Table 2

Examples of white noises with their Lévy exponent.

\begin{tabular}{lll}
\hline White noise & Parameters & Lévy exponent \\
\hline Gaussian & $\sigma^{2}>0$ & $-\frac{\sigma^{2} \xi^{2}}{2}$ \\
Laplace & $\sigma^{2}>0$ & $-\log \left(1+\frac{\sigma^{2} \xi^{2}}{2}\right)$ \\
Cauchy & $c>0$ & $-c|\xi|$ \\
Gauss-Poisson & $\lambda, \sigma^{2}>0$ & $\lambda\left(\mathrm{e}^{-\frac{\sigma^{2} \xi^{2}}{2 \lambda}}-1\right)$ \\
Laplace-Poisson & $\lambda, \sigma^{2}>0$ & $\lambda\left(\frac{1}{1+\frac{\sigma^{2} \xi^{2}}{2 \lambda}}-1\right)$ \\
Cauchy-Poisson & $\lambda, c>0$ & $\lambda\left(\mathrm{e}^{-\frac{c|\xi|}{\lambda}}-1\right)$ \\
\hline
\end{tabular}

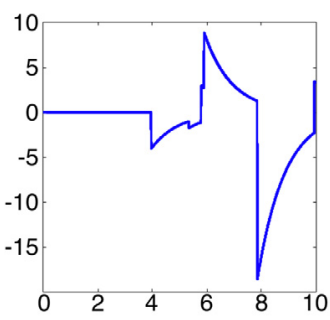

(a) Poisson, $\lambda=0.5$

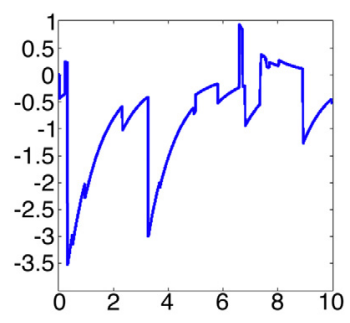

(b) Poisson, $\lambda=3$

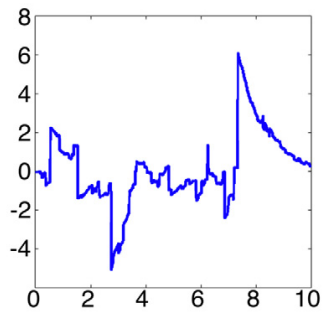

(c) Poisson, $\lambda=100$

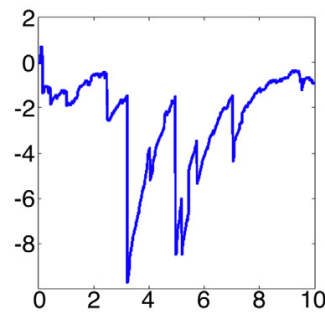

(d) Cauchy, $\lambda \rightarrow \infty$

Fig. 2. Processes generated by $\mathrm{D}+\alpha \mathrm{I}, \alpha=0.1$, so that $s=(\mathrm{D}+\alpha \mathrm{I})^{-1} w$. In (a)-(c), $w$ is a Poisson noise with Cauchy jumps, with increasing $\lambda$. In (d), $w$ is a Cauchy white noise.

The main purpose of the simulations is to give a visual illustration of our main result (Theorem 5). We shall see that typical values of $\lambda=100$ for $d=1$ (Section 6.1) and $\lambda=50$ for $d=2$ (Section 6.2) are sufficient to generate generalized random processes that are virtually indistinguishable from their Lévy counterparts. The next step will be to investigate the speed of convergence in (61), which would give mathematical guarantees for the convergence result. We postpone this theoretical question to future work. We however observe that the choice of the Lévy white noise qualitatively impact the approximation. Non-Gaussian white noises have a compound Poisson part, resulting in the presence of jumps [21]. A faithful approximation of the corresponding generalized Lévy process by generalized compound Poisson processes is therefore expected to be obtained with a lower value of $\lambda$ when the jumps are dominating.

\subsection{Simulations in 1-D}

We illustrate two families of 1-D processes, as given by

- $(\mathrm{D}+\alpha \mathrm{I}) s=w$, with parameter $\alpha>0$;

- $\mathrm{D} s=w$.

All the processes are plotted on the interval $[0,10]$. We show in Fig. 2 a Cauchy process generated by $\mathrm{D}+\alpha \mathrm{I}$. In Fig. 3 and 4, we show a Gaussian and a Laplace process, respectively. Both of them are whitened by $D$. In all cases, we first plot the processes generated with an appropriate Poisson noises with increasing values of $\lambda$. Then, we show the processes obtained from the corresponding Lévy white noise.

Interestingly, we observe that the processes obtained with Poisson noises of small $\lambda$ in Figs. 3 and 4 are very similar. However, their asymptotic processes (large $\lambda$ ) differ, as expected from the fact that they converge to processes obtained from different Lévy white noises. Moreover, for intermediate values of $\lambda$ (typically, $\lambda=3$ in this case), the Laplace-Poisson process is visually much closer to its Laplace limit than the Gauss-Poisson process from its Gaussian limit. We empirically deduce that generalized compound Poisson processes approximate sparse processes better than Gaussian processes, as discussed above. 


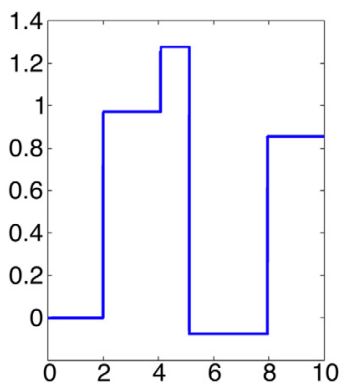

(a) Poisson, $\lambda=0.5$

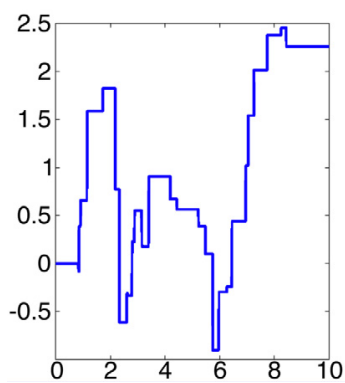

(b) Poisson, $\lambda=3$

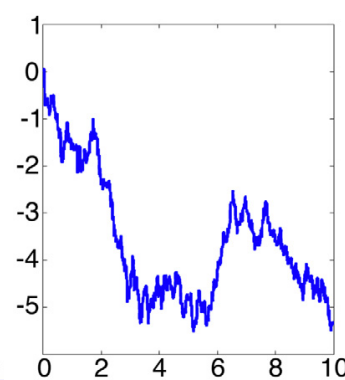

(c) Poisson, $\lambda=100$

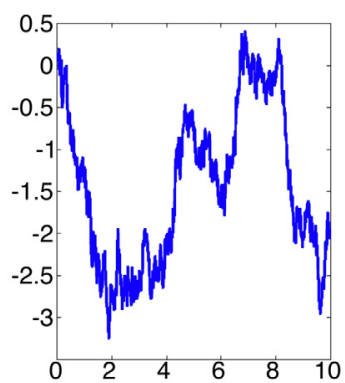

(d) Gaussian, $\lambda \rightarrow \infty$

Fig. 3. Processes whitened by D. In (a)-(c), $w$ is a Poisson noise with Gaussian jumps, with increasing $\lambda$. In (d), $w$ is a Gaussian white noise.

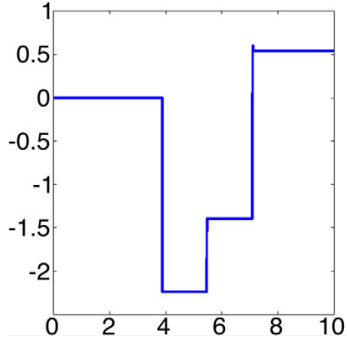

(a) Poisson, $\lambda=0.5$

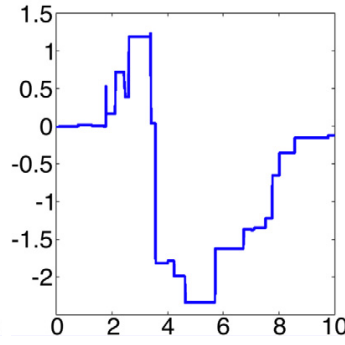

(b) Poisson, $\lambda=3$

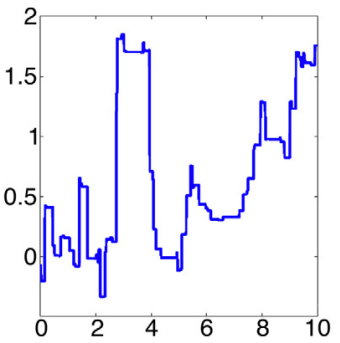

(c) Poisson, $\lambda=100$

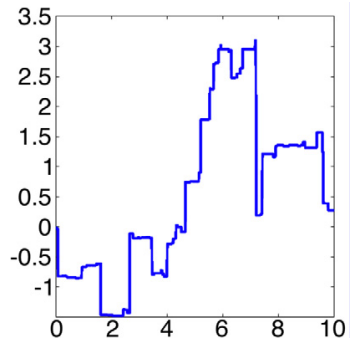

(d) Laplace, $\lambda \rightarrow \infty$

Fig. 4. Processes generated by $\mathrm{D}$, so that $s=\mathrm{D}^{-1} w$. In (a)-(c), $w$ is a Poisson noise with Laplace jumps, with increasing $\lambda$. In (d), $w$ is a Laplace white noise.

\subsection{Simulations in 2-D}

We illustrate three families of 2-D processes $s$, given as

- $\mathrm{D}_{x} \mathrm{D}_{y} s=w$

- $\left(\mathrm{D}_{x}+\alpha \mathrm{I}\right)\left(\mathrm{D}_{y}+\alpha \mathrm{I}\right) s=w$, with parameter $\alpha>0$;

- $(-\Delta)^{\gamma / 2} s=w$, with parameter $\gamma>0$.

We represent our 2-D examples in two ways: first as an image, with gray levels that correspond to the amplitude of the process (lowest value is dark, highest value is white); second as a 3-D plot. All processes are plotted on $[0,10]^{2}$. In Figs. 5 and 6 , we show a Gaussian process with $D$ as whitening operator. A Gaussian process generated by the fractional Laplacian $(-\Delta)^{\frac{\gamma}{2}}$ is illustrated in Figs. 7 and 8. Finally, we plot in Figs. 9 and 10 a Laplace process generated by $\left(\mathrm{D}_{x}+\alpha \mathrm{I}\right)\left(\mathrm{D}_{y}+\alpha \mathrm{I}\right)$. We always first show the process generated with an appropriate Poisson noise with increasing $\lambda$ and then plot the processes obtained from the corresponding Lévy white noise.

\section{Conclusion}

Our main result in this work is the proof that any generalized Lévy process $s=\mathrm{L}^{-1} w$ is the limit in law of generalized Poisson processes obeying the same equation, but where $w$ corresponds to an appropriate impulsive Poisson noises. In addition, we showed that generalized Poisson processes are random L-splines. In the asymptotic regime, generalized Lévy processes can thus conveniently be described using splines.

Theorem 1 is interesting in practice as it provides a new way of generating approximations of broad classes of sparse processes $s=\mathrm{L}^{-1} w$ as soon as one is able to generate the infinitely divisible random variable that 


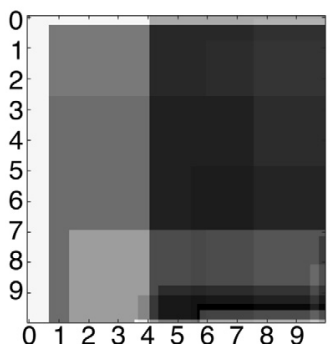

(a) Poisson, $\lambda=0.1$

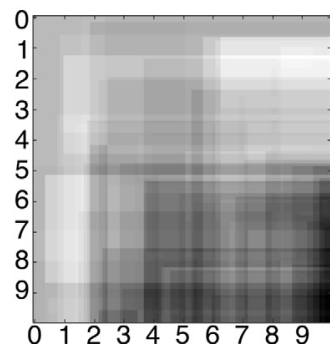

(b) Poisson, $\lambda=1$

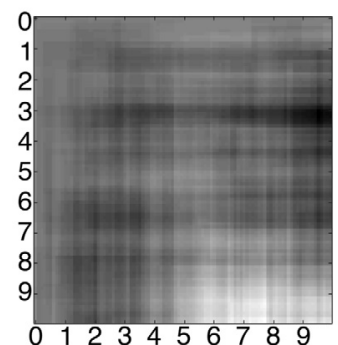

(c) Poisson, $\lambda=50$

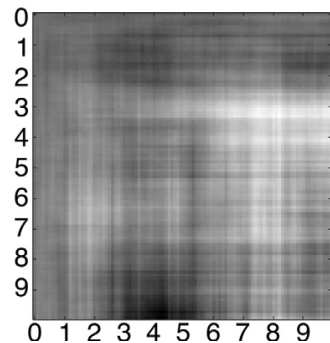

(d) Gaussian, $\lambda \rightarrow \infty$

Fig. 5. Processes generated by $\mathrm{D}$, so that $s=\mathrm{D}^{-1} w$. In (a)-(c), $w$ is a Poisson noise with Gaussian jumps, with increasing $\lambda$. In (d), $w$ is a Gaussian white noise.

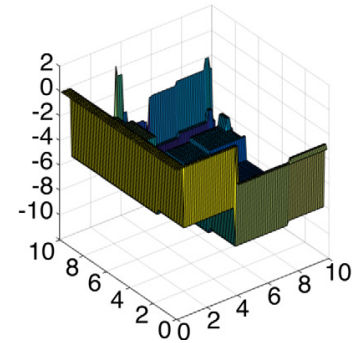

(a) Poisson, $\lambda=0.1$

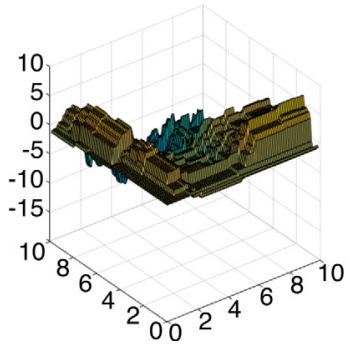

(b) Poisson, $\lambda=1$

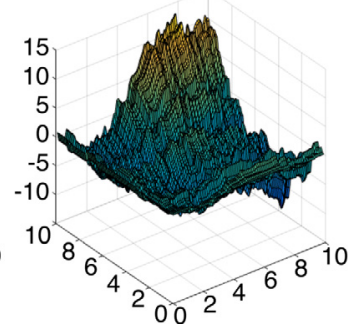

(c) Poisson, $\lambda=50$

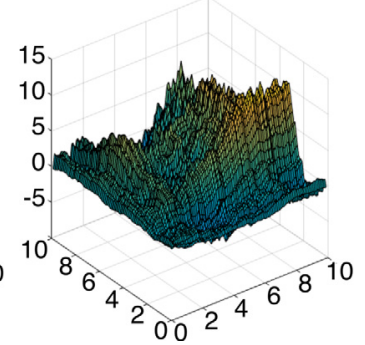

(d) Gaussian, $\lambda \rightarrow \infty$

Fig. 6. 3-D representation of processes generated by $\mathrm{D}$, so that $s=\mathrm{D}^{-1} w$. In (a)-(c), $w$ is a Poisson noise with Gaussian jumps, with increasing $\lambda$. In (d), $w$ is a Gaussian white noise.

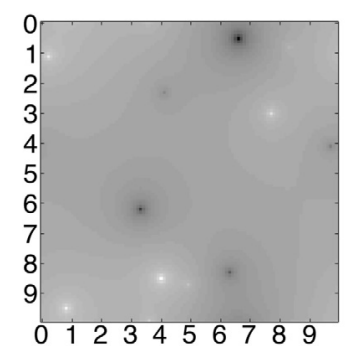

(a) Poisson, $\lambda=0.1$

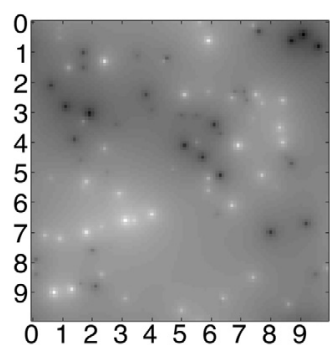

(b) Poisson, $\lambda=1$

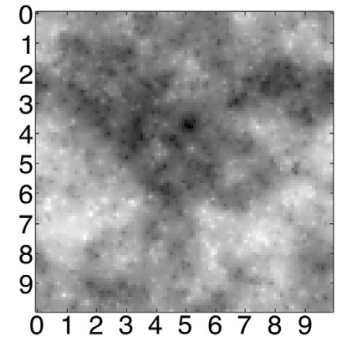

(c) Poisson, $\lambda=50$

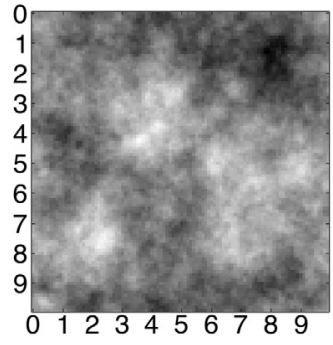

(d) Gaussian, $\lambda \rightarrow \infty$

Fig. 7. Processes generated by $(-\Delta)^{\frac{\gamma}{2}}, \gamma=1.5$, so that $s=\left((-\Delta)^{\frac{\gamma}{2}}\right)^{-1} w$. In (a)-(c), w is a Poisson noise with Gaussian jumps, with increasing $\lambda$. In (d), $w$ is a Gaussian white noise.

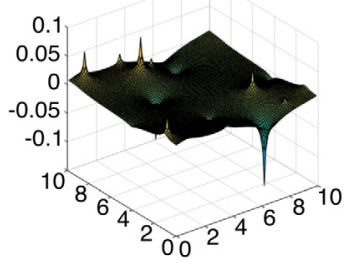

(a) Poisson, $\lambda=0.1$

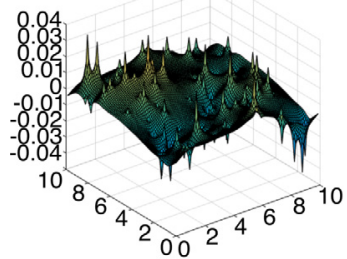

(b) Poisson, $\lambda=1$

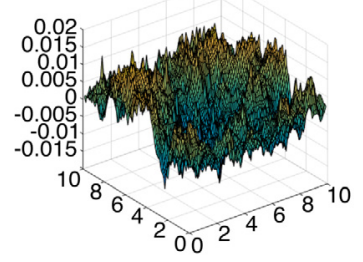

(c) Poisson, $\lambda=50$

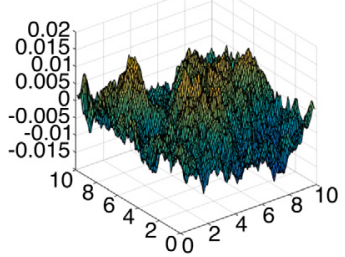

(d) Gaussian, $\lambda \rightarrow \infty$

Fig. 8. 3-D representation of processes generated by $(-\Delta)^{\frac{\gamma}{2}}, \gamma=1.5$, so that $s=\left((-\Delta)^{\frac{\gamma}{2}}\right)^{-1} w$. In $($ a $)-($ c), $w$ is a Poisson noise with Gaussian jumps, with increasing $\lambda$. In (d), $w$ is a Gaussian white noise.

drives the white noise $w$. This confirms the remarkable intuition that Bode and Shannon enunciated decades before the formulation of the mathematical tools needed to prove their claims. A precise estimation of the speed of convergence of generalized Poisson processes should moreover give theoretical guarantees that the 


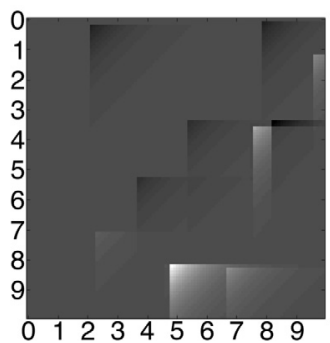

(a) Poisson, $\lambda=0.1$

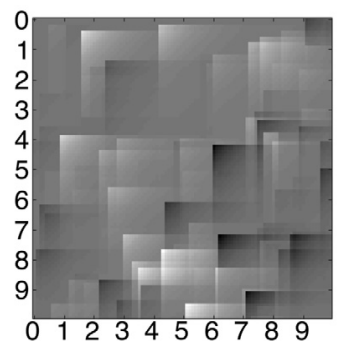

(b) Poisson, $\lambda=1$

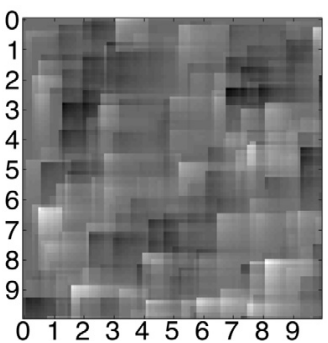

(c) Poisson, $\lambda=50$

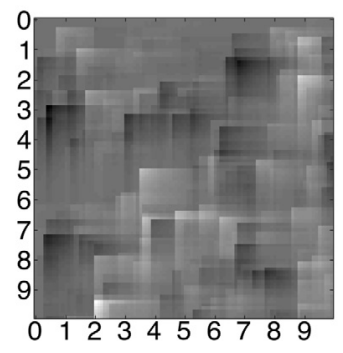

(d) Laplace, $\lambda \rightarrow \infty$

Fig. 9. Processes generated by $\left(\mathrm{D}_{x}+\alpha \mathrm{I}\right)\left(\mathrm{D}_{y}+\alpha \mathrm{I}\right), \alpha=0.1$, so that $s=\left(\left(\mathrm{D}_{x}+\alpha \mathrm{I}\right)\left(\mathrm{D}_{y}+\alpha \mathrm{I}\right)\right)^{-1} w$. In $(\mathrm{a})-(\mathrm{c}), w$ is a Poisson noise with Laplace jumps, with increasing $\lambda$. In $(\mathrm{d}), w$ is a Laplace white noise.

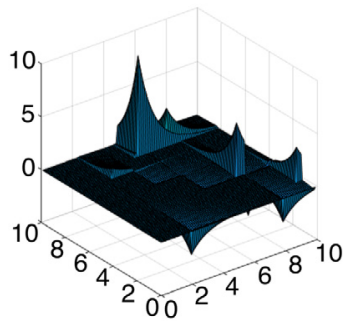

(a) Poisson, $\lambda=0.1$

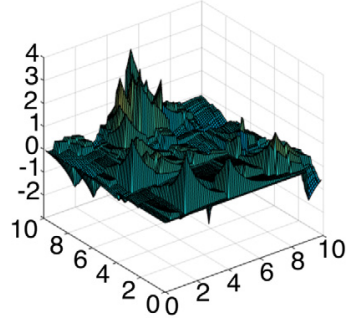

(b) Poisson, $\lambda=1$

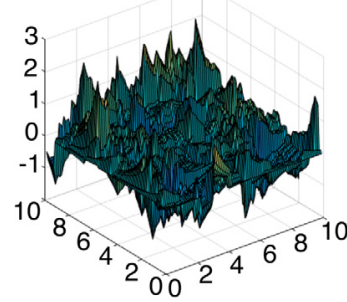

(c) Poisson, $\lambda=50$

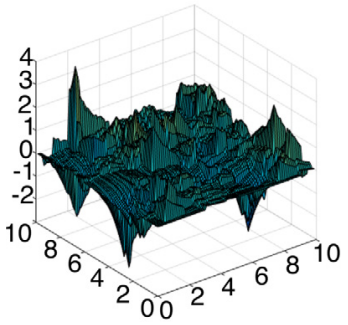

(d) Laplace, $\lambda \rightarrow \infty$

Fig. 10. 3-D representation of processes generated by $\left(\mathrm{D}_{x}+\alpha \mathrm{I}\right)\left(\mathrm{D}_{y}+\alpha \mathrm{I}\right), \alpha=0.1$, so that $s=\left(\left(\mathrm{D}_{x}+\alpha \mathrm{I}\right)\left(\mathrm{D}_{y}+\alpha \mathrm{I}\right)\right)^{-1} w$. In $(\mathrm{a})-(\mathrm{c})$, $w$ is a Poisson noise with Laplace jumps, with increasing $\lambda$. In (d), $w$ is a Laplace white noise.

approximation and the original process $s$ are statistically identical. We keep this research endeavors for future work.

\section{Acknowledgments}

This work was supported in part by the European Research Council under Grant H2020-ERC (ERC grant agreement No. 692726 - GlobalBioIm), and in part by the Swiss National Science Foundation under Grant 200020_162343/1.

\section{References}

[1] H.W. Bode, C.E. Shannon, A simplified derivation of linear least square smoothing and prediction theory, Proc. IRE 38 (4) (1950) 417-425.

[2] M. Unser, Splines: a perfect fit for signal and image processing, IEEE Signal Process. Mag. 16 (6) (1999) $22-38$.

[3] I. Schoenberg, Cardinal Spline Interpolation, SIAM, Philadelphia, PA, 1973.

[4] W. Madych, S. Nelson, Polyharmonic cardinal splines, J. Approx. Theory 60 (2) (1990) 141-156.

[5] M. Schultz, R. Varga, L-splines, Numer. Math. 10 (4) (1967) 345-369.

[6] M. Unser, T. Blu, Fractional splines and wavelets, SIAM Rev. 42 (1) (2000) 43-67.

[7] M. Unser, P.D. Tafti, Stochastic models for sparse and piecewise-smooth signals, IEEE Trans. Signal Process. 59 (3) (2011) 989-1006.

[8] M. Vetterli, J. Kovačević, V.K. Goyal, Foundations of Signal Processing, Cambridge University Press, 2014.

[9] T. Blu, M. Unser, Self-similarity: part II-optimal estimation of fractal processes, IEEE Trans. Signal Process. 55 (4) (2007) 1364-1378.

[10] B. Mandelbrot, The Fractal Geometry of Nature, W. H. Freeman and Co., San Francisco, California, 1982.

[11] B. Mandelbrot, J.V. Ness, Fractional Brownian motions, fractional noises and applications, SIAM Rev. 10 (4) (1968) 422-437.

[12] B. Pesquet-Popescu, J.L. Véhel, Stochastic fractal models for image processing, IEEE Signal Process. Mag. 19 (5) (2002) $48-62$.

[13] D. Mumford, A. Desolneux, Pattern Theory: The Stochastic Analysis of Real-World Signals, A.K. Peters, Ltd., Natick, MA, 2010.

[14] D. Mumford, B. Gidas, Stochastic models for generic images, Quart. Appl. Math. 59 (1) (2001) 85-112. 
[15] A. Srivastava, A. Lee, E. Simoncelli, S.-C. Zhu, On advances in statistical modeling of natural images, J. Math. Imaging Vision 18 (1) (2003) 17-33.

[16] C. Nikias, M. Shao, Signal Processing with Alpha-Stable Distributions and Applications, Wiley-Interscience, 1995.

[17] J. Ward, J. Fageot, M. Unser, Compressibility of symmetric- $\alpha$-stable processes, in: Proceedings of the Eleventh International Workshop on Sampling Theory and Applications (SampTA'15), Washington DC, USA, May 25-29, 2015, pp. 236-240.

[18] J. Fageot, M. Unser, J. Ward, The n-term approximation of periodic generalized Lévy processes, arXiv preprint, arXiv: 1702.03335, 2017.

[19] H. Ghourchian, A. Amini, A. Gohari, How compressible are sparse innovation processes?, arXiv preprint, arXiv:1703.09537, 2017.

[20] M. Unser, P.D. Tafti, An Introduction to Sparse Stochastic Processes, Cambridge University Press, 2014.

[21] K. Sato, Lévy Processes and Infinitely Divisible Distributions, vol. 68, Cambridge University Press, Cambridge, 2013.

[22] L. Schwartz, Théorie des distributions, Hermann, 1966.

[23] K. Itô, Stationary random distributions, Kyoto J. Math. 28 (3) (1954) 209-223.

[24] I. Gelfand, Generalized random processes, Dokl. Akad. Nauk SSSR 100 (1955) 853-856.

[25] I. Gelfand, N. Vilenkin, Generalized Functions, vol. 4: Applications of Harmonic Analysis, Academic Press, New York, London, 1964.

[26] K. Itô, Foundations of Stochastic Differential Equations in Infinite Dimensional Spaces, vol. 47, SIAM, 1984.

[27] E. Bostan, U. Kamilov, M. Nilchian, M. Unser, Sparse stochastic processes and discretization of linear inverse problems, IEEE Trans. Image Process. 22 (7) (2013) 2699-2710.

[28] S. Aziznejad, J. Fageot, M. Unser, Wavelet analysis of the Besov regularity of Lévy white noises, arXiv preprint, arXiv: $1801.09245,2018$.

[29] J. Fageot, Gaussian versus Sparse Stochastic Processes: Construction, Regularity, Compressibility, EPFL thesis no. 7657, Swiss Federal Institute of Technology Lausanne (EPFL), April 24, 2017, 231 p.

[30] E. Perrin, R. Harba, C. Berzin-Joseph, I. Iribarren, A. Bonami, Nth-order fractional Brownian motion and fractional Gaussian noises, IEEE Trans. Signal Process. 49 (5) (2001) 1049-1059.

[31] H. Biermé, A. Estrade, I. Kaj, Self-similar random fields and rescaled random balls models, J. Theoret. Probab. 23 (4) (2010) 1110-1141.

[32] H. Biermé, L. Moisan, R. Richard, A turning-band method for the simulation of anisotropic fractional Brownian fields, J. Comput. Graph. Statist. 24 (3) (2015) 885-904.

[33] A. Lodhia, S. Sheffield, X. Sun, S. Watson, Fractional Gaussian fields: a survey, Probab. Surv. 13 (2016) 1-56.

[34] P.D. Tafti, M. Unser, Fractional Brownian vector fields, Multiscale Model. Simul. 8 (5) (2010) 1645-1670.

[35] J. Fageot, E. Bostan, M. Unser, Wavelet statistics of sparse and self-similar images, SIAM J. Imaging Sci. 8 (4) (2015) 2951-2975.

[36] J. Fageot, M. Unser, Scaling limits of solutions of stochastic differential equations driven by Lévy white noises, J. Theoret. Probab. (2018) 1-24, https://doi.org/10.1007/s10959-018-0809-1.

[37] G. Samorodnitsky, M. Taqqu, Stable Non-Gaussian Processes: Stochastic Models with Infinite Variance, Chapman and Hall, 1994.

[38] H. Biermé, M. Meerschaert, H.-P. Scheffler, Operator scaling stable random fields, Stochastic Process. Appl. 117 (3) (2007) $312-332$.

[39] S. Koltz, T. Kozubowski, K. Podgorski, The Laplace Distribution and Generalizations, Birkhauser, Boston, MA, 2001.

[40] B. Grigelionis, Student's T-Distribution and Related Stochastic Processes, Springer, 2013.

[41] D. Applebaum, Lévy Processes and Stochastic Calculus, Cambridge University Press, 2009.

[42] P. Brockwell, Lévy-driven CARMA processes, Ann. Inst. Statist. Math. 53 (1) (2001) 113-124.

[43] P. Brockwell, J. Hannig, CARMA $(p, q)$ generalized random processes, J. Statist. Plann. Inference 140 (12) (2010) 3613-3618.

[44] R. Dalang, J. Walsh, The sharp Markov property of Lévy sheets, Ann. Probab. (1992) 591-626.

[45] A. Durand, S. Jaffard, Multifractal analysis of Lévy fields, Probab. Theory Related Fields 153 (1-2) (2012) 45-96.

[46] M. Vetterli, P. Marziliano, T. Blu, Sampling signals with finite rate of innovation, IEEE Trans. Signal Process. 50 (6) (2002) 1417-1428.

[47] J. Fageot, J. Ward, M. Unser, Interpretation of continuous-time autoregressive processes as random exponential splines, in: Proceedings of the Eleventh International Workshop on Sampling Theory and Applications (SampTA'15), Washington DC, USA, May 25-29, 2015, pp. 231-235.

[48] M. Unser, T. Blu, Cardinal exponential splines: part I-theory and filtering algorithms, IEEE Trans. Signal Process. 53 (4) (2005) 1425-1438.

[49] M. Unser, T. Blu, Self-similarity: part I-splines and operators, IEEE Trans. Signal Process. 55 (4) (2007) $1352-1363$.

[50] D. Van De Ville, T. Blu, M. Unser, Isotropic polyharmonic B-splines: scaling functions and wavelets, IEEE Trans. Image Process. 14 (11) (2005) 1798-1813.

[51] H. Biermé, O. Durieu, Y. Wang, Generalized random fields and Lévy's continuity theorem on the space of tempered distributions, arXiv preprint, arXiv:1706.09326, 2017.

[52] A. Pietsch, Nuclear Locally Convex Spaces, Springer, 1972.

[53] F. Trèves, Topological Vector Spaces, Distributions and Kernels, Academic Press, New York, London, 1967.

[54] A. Grothendieck, Produits tensoriels topologiques et espaces nucléaires, in: Séminaire Bourbaki, vol. 2, Soc. Math. France, Paris, 1995.

[55] D. Mushtari, Probabilities and Topologies on Linear Spaces, vol. 1, Kazan Mathematics Foundation, 1996.

[56] R. Minlos, Generalized random processes and their extension in measure, Tr. Mosk. Mat. Obs. 8 (1959) 497-518.

[57] X. Fernique, Processus linéaires, processus généralisés, Ann. Inst. Fourier 17 (1967) 1-92. 
[58] P. Boulicaut, Convergence cylindrique et convergence étroite d'une suite de probabilités de Radon, Z. Wahrsch. Verw. Gebiete 28 (1973/74) 43-52.

[59] J. Fageot, A. Amini, M. Unser, On the continuity of characteristic functionals and sparse stochastic modeling, J. Fourier Anal. Appl. 20 (2014) 1179-1211.

[60] M. Unser, P.D. Tafti, Q. Sun, A unified formulation of Gaussian versus sparse stochastic processes-part I: continuousdomain theory, IEEE Trans. Inform. Theory 60 (3) (2014) 1945-1962.

[61] R. Horn, Quadratic forms in harmonic analysis and the Bochner-Eberlein theorem, Proc. Amer. Math. Soc. 52 (1) (1975) 263-270. 